\title{
Development status of LASER Cladding and the new metallic alloys
}

Teresa Morgado ${ }^{1,2,3}$, Catarina Valente ${ }^{1}$

1. DEMI - Mechanical and Industrial Engineering Department, FCT NOVA - Faculty of Sciences and Technology, Universidade Nova de Lisboa, Lisboa, Portugal, email: c.valente@campus.fct.unl.pt; t.morgado@fct.unl.pt

2. UNIDEMI - Research and Development Unit for Mechanical and Industrial Engineering Universidade Nova de Lisboa, Lisboa, Portugal

3. IPT - Polytechnic Institute of Tomar, Tomar, Portugal

\begin{abstract}
LASER cladding is a technique which has as main purpose to improve mechanical properties of materials surface with the addition of thin layers of other materials. Cladding/coating/addition material can be transferred to the substrate by powder injection, wire feeding or paste feeding and by the method of pre-placing the powders on the substrate.

LASER cladding as a technique has only about 40 years. Is not yet a current technology and more work is needed, namely in the characterization of mechanical properties of new alloys used as base or cladding materials in this process.

In this paper will be done initially an historical approach, followed by a description of the principles of the LASER cladding, a description of the applicability of the process and its advantages and limitations and the paper will end with a state of the art about the advances in new metallic alloys. So, this paper presents a review and the state of the art of the technological process and will be a contribution for the divulgation of this recent technique.
\end{abstract}

Keywords: LASER Cladding; Manufacturing Process; Metallic Alloys; Innovation; State of the Art

\section{Introduction}

LASER cladding process which emerged with the purpose of improving the characteristics of metallic components' surfaces, mainly regarding the corrosion and wear resistance, came to take the advantages of the LASER created by Maiman in the 60 s and was considered, at that time, a great invention for science. LASER would become an answer to a huge variety of scientific questions, until then unanswered for years, as in the case of the materials processing area that has rapidly developed in the 70s, as soon as the power and efficiency of commercial LASER increased [1],[2]. 
The application of LASER technology for material addition/deposition processes came to improve the precision of material deposition, to reduce the dilution of the substrate and the temperature increase of the component to be coated and also, the utilization of a LASER beam, does not cause unfavourable alterations in the mechanical properties of the melt pool [3].

Although, in the last years some articles about this process have emerged, namely from Liu et al. [4], Weng et al. [5], Birger et al. [6] or Zhong and Liu [7]. Its utilization is recent, presents many potentialities and is still being implemented and disclosed in research centres, technological centres and in a few industries, all over the world. Thus, this article aims to contribute with the state of the art of the LASER cladding process, boosting the divulgation of the technical and scientific knowledge.

\section{History of LASER Cladding Process}

LASER cladding process was introduced and patented by Daniel S. Gnanamuthu in 1976, in USA [2],[8]. The reason for its creation, was improving the quality of components' surfaces, overtaking the already known disadvantages in traditional processes as TIG - Tungsten Inert Gas welding, also known as GTAW - Gas Tungsten Arc Welding, plasma spray or HVOF - High Velocity Oxy Fuel, being these disadvantages the high dilution of the substrate material into the coating/cladding, the large increase of temperature imposed by the process resulting in distortions in the parts, the low precision in material deposition, porosities, micro-cracks, bond defects and problems in the adherence to the substrate [3],[9].

The pre-placed LASER cladding method, with the pre-placing of the cladding material on the substrate, was initially used by Gnanamuthu at Rockwell International Corporation, in California, to investigate the feasibility of the process in the application of dense ceramic cladding on metallic components. One of the research groups that had a significant impact in the development of this new technology, led by William M. Steen, introduced LASER cladding by powder injection. Another of the research groups, conducted by Jyoti Mazumder in the 80 s, contributed for the knowledge of possible principles of the process and also applied the technology to various metals and ceramics to have the chance to investigate their cladability potential and also their posterior corrosion and wear resistance. Note that, one of the considered definitions for cladability, that is a term related to cladding material, includes the ability of the material to form a continuous and high density clad, with a uniform or homogeneous microstructure, having a strong metallurgical bond to the substrate, with low dilution [2].

Still in the 1980s, LASER cladding has started to arouse industry's attention, having been identified as a process that presents great advantages in improving the corrosion and wear resistance of coatings over the conventional processes. First report of its use in industry occurred in 1981, at Rolls Royce, in the coating of turbine blades components made by Nimonic that would be used in RB-211 jet engine, having its utilization extended to the leading companies in the engine production sector at that time. In the automotive industry, LASER cladding has also started to be applied in the engine valve coating as in the case of Fiat, Toyota and Mercedes-Benz. Still in the same decade, the process was widely recognized by repair market, mainly in turbine blades repairing, both in USA and in Europe [2]. 
In the 90 s, a large number of rapid prototyping or layered manufacturing methods of three-dimensional structures, that already used the principles of the cladding process, were introduced and patented, doing now part of the commercial available solutions [2],[8].

\section{Principles of the LASER Cladding}

LASER cladding is considered a surface treatment technique with LASER. It is possible to distinguish three groups of techniques of surface treatment with LASER. The group of surface hardening techniques (or without surface melting techniques), the group of surface melting techniques and the group of techniques with surface melting and with addition material [10],[11]. In the case that is not possible for the base material to improve its mechanical properties on its own with melting or hardening techniques, it becomes necessary or cheaper to resort to the application of a layer of other material on the base material surface [10].

Within the techniques that allow the improvement of material properties by the composition alteration of the surface layer with addition of material and within the ones that have in common the formation of a melt pool in which the addition material is applied, is found LASER cladding technique that is then characterized [10]. The other techniques that belong to this group are LASER alloying and LASER dispersing that, unlike LASER cladding, are characterized by an intensive melting of the substrate where the material to be added to create the coating will be incorporated, interacting with the melt. On the other side, LASER cladding technique includes the melting of the addition/cladding material, minimizing the melting of the substrate material [3].

LASER cladding uses LASER as the heat source to the deposition of a thin layer of a certain material with desired properties on a certain substrate, that melts the cladding material surface and the substrate surface [2],[11]. The area to be cladded is heated by the absorption of energy delivered by the LASER beam. The heat input provided by a high-power LASER (in most industrial applications the most used power is above $5 \mathrm{~kW}$ ) beam is well confined and very intense, increasing thus the heating rate of the surface layer. The heated surface layer becomes quenched, after the passing of the LASER by diffusion of heat to the coldest part of the bulk. High rates of heating/cooling in the surface layer result in grain refinement and in the formation of metastable phases and/or in the alteration of the microstructures. LASER cladding process traditionally uses $\mathrm{CO}_{2}$ LASER and various types of Nd:YAG LASER, although, more recently, it has been betting on the use of fibre LASER [10],[12].

Cladding material can be transferred to the substrate by powder injection, wire feeding or paste feeding (methods of continuous application of the addition material during the cladding process) and by the method of pre-placing the powders of the material on the substrate [2],[10].

Pre-placed powder method corresponds to a two-stage method, beginning the first stage with the application of powder on the substrate. The powder should be mixed with a chemical binder, forming a distributed paste throughout the substrate, ensuring good bond between the cladding material and the substrate as the process takes place. The binder evaporates during the process, which may be the cause of some porosities in the clad layer. In the second stage, occurs the formation of a melt pool on the surface of the cladding material, being propagated subsequently to the interface with the substrate. 
Proceeding the heating, it is possible to cause the melt pool to extend to the substrate, creating a strong bond between the material added and the substrate [10],[11].

Relatively to the continuous feeding method during the process by powder injection, which only presents one stage, the melt pool forms initially on the substrate, being the cladding material injected, at the same time to that melt pool, melting itself. In the powders interaction with the LASER beam, powders suffer a heating before they are fed into the melt pool that is being created on the substrate. Reaching the melt pool that is created, the cladding material is going to melt, creating a strong bond between the deposited material and the substrate material. Is common the utilization of an inert gas flow as a mean of transportation of powder particles to the melt pool. In this method, instead of powder, the cladding material can come in wire form or even in the form of a paste constituted by the powders and a suitable binder that is being supplied during the process (allowing a previous formation of alloys that are going to be deposited), being however, this last one less common than the first two kinds of continuous material feeding (much less when compared to the powder injection - the most common) and for that less described in literature [10],[11],[13].

In relation to wire feeding, slight changes in wire position or in feeding velocity can cause significant alterations in the form and dimension of the melt pool, resulting in a hardly uniform and asymmetric clad, thus requiring greater accuracy during the process. The wire also causes a certain obstruction to the LASER, reducing the percentage of the LASER beam that reaches the surface of the base material, when comparing to the powder injection form of feeding, in which the LASER passes more easily through the stream of the particles. What also permits to enhance the advantage of adding the material in powder form is the possibility to vary the quantity of the elements present in the powder mixture, during their deposition, making possible the production of a wide variety of alloys [11].

Powder injection method has been shown as to be the most efficient and most used. There is already a big variety of materials that can be utilized as cladding material in LASER cladding by powder injection, usually forming layers with thickness ranging from 0.05 to $2 \mathrm{~mm}$ and widths of $0.4 \mathrm{~mm}$ [2],[10],[11].

To realize LASER cladding process, in case of a continuous material feeding method, is necessary a device capable of bringing the powder stream, the wire or the paste that is going to correspond to the cladding material, the shielding gas and the LASER heat source. All these valences are included in the LASER cladding heads [8].

Although, besides the LASER cladding head that allows the reach of the LASER to the surface to be treated, the total equipment to the LASER cladding execution contains two more essential components, a positioning system XYZ (positioning table of two axis) and a powder feeder as was demonstrated in Torres et al. work [14] of development and automation of the LASER cladding equipment with powder injection from the LASER Laboratory of IST, Lisbon University, used by CeFEMA - Center of Physics and Engineering of Advanced Materials. In this work was developed a control process to the LASER cladding equipment through the utilization of a personal computer. In Figure 1 is possible to visualize the control scheme of the equipment needed to execute the LASER cladding process. 


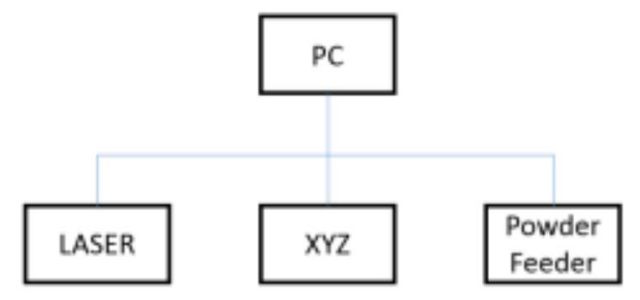

Figure 1-Control scheme of the LASER cladding equipment [14]

This automation, which was achieved through the development of the control process, had in mind the optimization of simultaneous utilization of the equipment components and so, allows the control of process parameters such as the overlapping of clads, number of clads, deposition rate, LASER power and powder feed rate. Controlling these parameters is essential to obtain quality coatings, since these will influence their mechanical properties and durability [14], [15].

\subsection{Applicability of the Process}

LASER cladding technology has a strong applicability in the surface coating sector, mainly in metallic surfaces, although, it has been arousing, more and more, the interest in the components repair and rapid prototyping sector [2],[16].

To show the sense of LASER cladding application, in response to the demand for an increase in efficiency and for a reduction in costs of aircraft gas turbines, cladding materials with high mechanical resistance and resistance to elevated temperatures are being used through the application of LASER cladding, as is the case of nickel-based super alloys. It is expected that with the improvements that are being operated in the new kinds of LASER, LASER cladding technology starts to represent a more important role in this gas turbine market and also in aerospace industry in general [2].

Coating obtained by LASER cladding also has other applications in terms of the coating for industrial components with a view to the production of surfaces that are resistant to abrasive, erosive and adhesive wear, corrosion resistant in liquid medium and resistant to oxidation and corrosion at high temperatures, as well. Some examples of products that received metallic coating by LASER cladding, getting the above mentioned characteristics, were parts from drilling tools, engine valve seats, hydraulic pump components and moulds, as well as coated tools with hardfacing coatings for their surface hardening (tools hardfacing) [2].

LASER cladding technology is also used in the repair of high value components, as is the case of some tools, turbine blades and components for military industry. As an example of something repaired by LASER cladding, there is an exterior surface, also called shell by Toyserkani et al. [2], made of high resistance aluminium-based alloys (7075 and 7175 alloys, not weldable), used in weapon components integrated in submarines that are subject to wear and damage caused by handling difficulties, by the weapon proper activity and also by the saltwater corrosive nature [2],[16].

In the rapid prototyping and tooling sector, LASER cladding application allows the manufacturing of complex components and tools in a short time with recourse to CAD models. The little need of machining and the use of CAD models contribute for such reductions in the manufacturing time. LASER 
Engineering Net Shaping ${ }^{\mathrm{TM}}$, a rapid metal prototyping process by LASER cladding developed in Sandia National Laboratories, USA, being this one of the names by which rapid prototyping process that utilizes LASER cladding technology is known, is being utilized, for example, for processing rotor blades [2],[16]. As an example of a recent research work about LASER cladding process there is Feng et al. work [17] in which Inconel 625 coatings fabricated by LASER cladding and by SMAW - Shielded Metal Arc Welding were analysed and compared, at room and elevated temperatures, in terms of their microstructure, hardness and their wear resistance. These coatings, obtained with an equipped system with a $10 \mathrm{~kW}$ high-power ytterbium fibre LASER denominated IPG YLS-10000, had as substrate gas turbine valves of martensitic steel COST $E$ with dimensions of $700 \times 400 \times 40 \mathrm{~mm}$. Results indicated that the LASER cladding coating made with Inconel 625 powders (spherical particles in the range of 75-100 $\mu \mathrm{m})$, when compared with the one obtained by SMAW, provided a finer grain, a large presence of strengthening elements as molybdenum (Mo) and niobium $(\mathrm{Nb})$ remained in solid solution matrix and a lower dilution of iron that came from the base material. It was also possible to determine that, when comparing the two processes, the coating made by LASER cladding had, at room temperature, a slightly higher hardness in the coating zone being much higher in the iron dilution zone and, at elevated temperatures, had also higher hardness in all the coating. At all the different temperatures, LASER cladded coating had a lower wear rate due to the lower iron dilution and higher hardness. Therefore, the authors concluded that the coating produced by LASER cladding was preferable, since it presented a better mechanical performance at room and elevated temperatures.

LASER cladding is also being study as a method for improving the performance of standard grade rail, at a wear and RCF - Rolling Contact Fatigue level, as described by Lewis et al. [18]. Diverse types of metals, chosen by their elevated hardness and proven or expected high resistance to wear and RCF, were used as the cladding material for the standard R260 grade rail discs, having these been tested against discs made from standard R8 steel, in a not cladded condition, used in wheels in contact with the rails.

Still considering the last years development of LASER cladding process, it is also being used, for example, to investigate its potential for repairing certain materials as the case of AerMet ${ }^{\circledR} 100$ steel as is described in Sun et al. research work [19], being this steel, a type of high resistance steel widely utilized in aerospace applications, subject to critical conditions, as in landing gears. These are steels that are expose to high stresses in service conditions, are sensitive to fracture and tend to have a limited toughness. In aircraft applications, the damages caused by impact and corrosion and the required structural integrity, make repair essential. The difficulty in repairing this kind of steels it is due to the fact that it is essential that this repair allows the structure to carry high stresses without any reduction in steels resistance to fatigue and fracture.

Tuominen, in his Ph.D. thesis titled Engineering Coating by Laser Cladding - The Study of Wear and Corrosion Properties, does a detailed description of the groups of materials most commonly used in the LASER cladding process in the field of coating and most commonly studied when subjected to the process in this coating field, both as base and coating/cladding materials. Relatively to the base materials, the author refers as main groups the Fe-based materials group including carbon-manganese 
(C-Mn) steels, alloy steels, stainless steels, tool steels and cast irons, the aluminium alloys group, the Ni-based superalloys strengthened (by precipitates, for example) group, the titanium and its alloys group, the magnesium alloys group and the cooper and its alloys group. Regarding the coating/cladding materials, he refers as main groups the Co-based hardfacing alloys group, the Ni-based alloys group, the Fe-based materials group (namely, stainless steels, tool steels and Fe-based hardfacing alloys), the Cu-based alloys group, the Al-based coatings group, the Ti-based coatings group and also the group of metallic matrix composites, the group of functionally graded materials, the group of solid lubricants, the group of rare-earth element additions and the group of intermetallics [20].

In the case of rapid prototyping using LASER cladding technology that, among other names, is known by LASER Engineering Net Shaping or three-dimensional LASER cladding, the base material is usually metallic and serve as the construction base for the component to be built. Besides this, generally, base material corresponds to the same material that is going to be deposited (cladding material), normally by a continuous powder feeding, to produce the component, except in the cases that the use of a base material different from the cladding material is convenient to allow a better heat dissipation. Some examples of materials already tested in this field of LASER cladding that allows the rapid production of components encompass aluminium, stainless steels as the $304 \mathrm{~L}$ and the 316 , tool steels as the $\mathrm{H} 13$ and nickel-based alloys as the Inconel 600, 625, 690 and the 718 [2],[21].

\subsection{Advantages and Limitations of the Process}

LASER cladding technology presents advantages since material properties, such as wear and corrosion resistance and also hardness, can be improved. These represent some of the properties that are advantageous when increased, for example, in mechanical components subject to aggressive environmental conditions, to high thermal cycles or in mechanical components that are exposed to corrosive environments. In addition to these properties, the components' lifetime is also increased with the application of the LASER cladding. LASER cladding also presents advantages when used as a rapid prototyping technique or as a repair technique. Its utilization as a rapid prototyping technic allows the production of the components in a layer-by-layer way with a combination of characteristics that are considered unique in this process of LASER cladding as a rapid prototyping technique, both in case of the pre-placed powder method as in the continuous material feeding method in powder form. Among these characteristics are found the achievement of a homogeneous structure, good mechanical properties, as it already is a characteristic of the materials subject to LASER cladding, and the rapid production of components with complex structures that acquire a shape very close to the desired one at the end. Comparing to other existing rapid prototyping techniques, as the case of 3D printing, LASER cladding presents more quality in metallic components production. In what concerns to the components repair, LASER cladding technology, mainly with powder injection, has the advantage of allowing the repair of components (mainly tools, in their critical contact surfaces), improving their life time and in some cases actually saving high value components that, otherwise, would have to be replaced, which would cause elevated costs. Represents an alternative to the most used welding processes that produce high temperatures in the materials to be repaired and present a destructive character [2],[15]. 
It should be noted that some of the competitive characteristics of this process have their origin in the use of the LASER technology. The utilization of the LASER beam as heat source introduce advantages in relation to the traditional heat sources, namely [10],[12]:

- the energy source can be well controlled due to the nature of the LASER beam, which is confined and narrow;

- allows to perform very localized surface treatments;

- the heat input is low, resulting in minimal thermal distortion;

- allows the achievement of fine microstructure due to the high heating and cooling rates;

- permits a non-contact treatment, not occurring wear of tools or force application in the parts;

- the depth of penetration of the LASER is well defined.

Besides these benefits brought to LASER cladding process by the LASER utilization, the process has other unique advantages such as a minimal dilution of the substrate, a strong fusion bond created between the substrate and the cladding material, a minimal thermal distortion of the materials, a greater ease of preventing porosities and the possibility of achieving a homogeneous distribution of the elements, mainly in the case of powder injection method [10],[12].

It's worth noting that the characteristics of the components' surface improved with the process, which are visible in the case of surfaces treated by LASER cladding in coating or repair applications (despites the also good characteristics obtained by components built through rapid prototyping by LASER cladding), are due to a reduced dilution that occurs between the substrate and the cladding material, which allows the preservation of the excellent mechanical properties of the coating produced. However, this improvement of surface characteristics it is not only due to the metallurgical characteristics of the coating, it also depends on the thermal cycles applied during the process. The high heating and cooling rates to what the components are subject during the process will result in a grain refinement, which leads to an increase in mechanical resistance of the materials [2],[15].

Although, despite of the advantages presented above, the process has, at the same time, some limitations. Disturbances in the process may result in significant changes in the clad quality, even when the operational conditions are kept. This low reproducibility, observed in the significant changes, derive from the high sensibility of LASER cladding to small alterations in operational parameters such as LASER power, scanning speed and feeding rate, and it can also result from unexpected perturbations that occur during the LASER cladding as the case of variations in absorptivity. Elevated cost of investment in LASER as well as low efficiency of the LASER sources are also shown to be LASER cladding limitations in the application of coatings, in the repair area and also in the rapid prototyping area. With continuous technological development in LASER sector and in sophisticated programs to control the process, LASER cladding reveals a great industrial potential for metallic coating (which includes the repair potential), and more recently, in rapid prototyping applications, existing already development of autonomous system of LASER cladding for rapid prototyping that dispenses an expensive formation of qualified personnel for this recent technology, making it more competitive in relation to other processes like 3D printing because of the reduction in its costs [2]. 


\section{Advances in new metallic alloys}

As it was previously referred, LASER cladding includes the deposition of the feedstock material in order to create strong metallurgical bonds between the substrate and the cladding material itself, minimizing, at the same time, the mixture of the elements from the substrate and the cladding material [3].

As examples of some promising alloys that are being studied, with published documentation, as substrate material treated by LASER cladding or as cladding material applied by LASER cladding, there are magnesium alloys as substrate [4], nickel-based alloys [6] and titanium alloys, more specifically the Ti-Ta alloys [15], as cladding materials.

Presently, LASER cladding of magnesium alloys become a new area of interest in terms of research and, in the review article elaborated by Liu et al. [4], various studies are mentioned about the LASER cladding on magnesium alloys (working as substrate), as the case of studies that approach the optimization of the process parameters to this kind of alloys (LASER power, scanning velocity, beam focal position, feeding method, etc.) and the selection of the cladding materials that are going to be deposited on the substrate of magnesium alloys. In this review made in this article, was also summarized the recent development status of the application of LASER cladding on these alloys, were identified problems related to the application of LASER cladding on these alloys and their viable solutions and was identified the future tendency of LASER cladding process on magnesium alloys. These alloys are presented as one of the most promising lightweight structural materials and hence the interest in their improvement for other applications beyond those already recognized. Treating these alloys by LASER cladding is justified by their characteristics of low corrosion and wear resistance that they present and limit their application in wet and salty environment and that, however can be improved with the addition of coatings. From some studies referenced in this review work, it was concluded that, for example, from the studied materials to clad/coat magnesium alloys, besides the traditional metallic materials (Al-based alloys), novel ternary alloys as the case of $\mathrm{Ni}_{60.16} \mathrm{Zr}_{33.84} \mathrm{Al}_{6.0}$ alloy, amorphous alloys as the $\mathrm{Zr}_{65} \mathrm{Al}_{7.5} \mathrm{Ni}_{10} \mathrm{Cu}_{17.5}$ alloy and high entropy alloys (HEA) like AlCoCrCuFeNi HEA alloy are widely used and exhibit apparent advantages in their utilization as cladding materials. As an example of the advantages obtained, in accordance to one of the investigation studies of Yue et al. [22], magnesium alloy specimens coated by amorphous alloys $\mathrm{Zr}_{65} \mathrm{Al}_{7.5} \mathrm{Ni}_{10} \mathrm{Cu}_{17.5}$ have proved to have a wear resistance thirteen times higher than that of the substrate, being the corrosion phenomenon also reduced.

In the Morgado et al. [15] work, was carried out a wear study of the innovative Ti-Ta alloys. To realize the study, alloys were deposited on a commercially pure grade 2 titanium substrate by LASER cladding process. Samples of Ti30Ta and Ti52Ta were studied. As LASER cladding belongs to the group of the LASER technologies, this process allows to work with elevated temperatures that is essential to elements with high melting points as the case of the titanium and with even higher melting points in the case of tantalum. The considered importance of these innovative alloys is related with their low Young Modulus (similar to the Young Modulus of the bone) and with titanium and tantalum biocompatibility, which are ideal characteristics to their application in biomedical industry. Taking this into account, to study their wear behaviour is crucial, since it influences their structural integrity. To be able to know this 
wear behaviour, as mentioned before, micro-scale wear tests were realized on the two kinds of Ti-Ta alloys and the results obtained, very similar for both alloys, demonstrated that the alloys volume wear increases linearly with the rotation of the wear steel ball, with the sliding distance and the time test. From the analysis of the ball, it was possible to determine that, for this test, only exists grooving abrasion and the volume of wear abrasion is higher for the alloy of lower hardness (Ti52Ta). Therefore, by their low Young Modulus, it is considered that both alloys can be used in biomedical applications, however, Ti52Ta presents a wear behaviour more compatible to what is expected for new implant alloys.

According to Birger et al. [6], in LASER cladding area a large number of studies have been published related with the investigation of the application of different cladding mixtures in relation to the base materials on which they are applied, being one of the studied mixtures the nickel-base mixtures applied as powder mixtures for coating by LASER cladding. As an example of the results demonstrated by one of these research studies (from Qingwu Meng, et al. [23]), the application of NiCoCrAlY alloy coatings on a substrate of Ti-6Al-4V alloy with a view to improve wear resistance of the titanium alloy substrate, resulted in a higher wear resistance comparing to that of the substrate and, besides that, resulted in high quality coatings, free of pores and cracks.

\section{Conclusions}

It is concluded that the LASER cladding is an emergent technology with great potentiality, both in the characterization of new alloys and in the improvement of well-known alloys. It is a quick and precise process, whose operational parameters include the LASER power, scanning velocity, beam focal position, feeding method, just to name some. This is, the LASER cladding depends on the material (substrate and cladding material) and on the LASER cladding equipment characteristics (LASER, 2/3 axis table, powder feeder, nozzle, etc.) and consequently on the control of its operational parameters. Is still necessary to collect more scientific knowledge to make its commercialization more feasible for small, medium and big companies.

\section{References}

[1] F. Gäbler, "Cladding rises to the top Diode LASER cutting», Industrial LASER Solutions for Manufacturing, vol. 31, n. 2, pp. 16-18, Mar/Abr 2016.

[2] E. Toyserkani, A. Khajepour, and S. F. Corbin, LASER Cladding. USA: CRC Press, 2005.

[3] V. K. Sarin, D. Mari, L. Lianes, e C. E. Nebel, Eds., Comprehensive Hard Materials - Volume 1 Hardmetals. UK: Elsevier, 2014.

[4] J. Liu, H. Yu, C. Chen, F. Weng, and J. Dai, «Research and development status of LASER cladding on magnesium alloys: A review», Opt. LASERS Eng., vol. 93, pp. 195-210, 2017.

[5] F. Weng, C. Chen, and H. Yu, «Research status of LASER cladding on titanium and its alloys: A review», Mater. Des., vol. 58, pp. 412-425, 2014.

[6] E. M. Birger, G. V. Moskvitin, A. N. Polyakov, and V. E. Arkhipov, «Industrial LASER cladding: current state and future», Weld. Int., vol. 25, n. 3, pp. 234-243, 2011. 
[7] M. Zhong and W. Liu, "LASER surface cladding: the state of the art and challenges», Proc. Inst. Mech. Eng. Part C J. Mech. Eng. Sci., vol. 224, n. 5, pp. 1041-1060, 2010.

[8] P. KHAZAN, M. STROH, H. FREIßE, and H. KÖHLER, «Manufacturing specimens made by direct powder deposition - Industrial LASER Solutions». [Online]. Available: http://www.industriallasers.com/articles/print/volume-29/issue-6/features/manufacturing-specimens-made-by-directpowder-deposition.html. [Accessed: 12-Fev-2017].

[9] J. Lambarri, J. Leunda, V. García Navas, C. Soriano, and C. Sanz, «Microstructural and tensile characterization of Inconel 718 LASER coatings for aeronautic components», Opt. LASERS Eng., vol. 51, n. 7, pp. 813-821, 2013.

[10] M. Schneider, «LASER CLADDING WITH POWDER: effect of some machining parameters on clad properties», Ph.D. Dissertation, Universiteit Twente, Nederland, 1998.

[11] J. M. S. P. Torres, «Improvement and Automatization of a LASER Cladding System» in Portuguese, Master Thesis, FCT, UNL - Universidade NOVA de Lisboa, Portugal, 2015.

[12] V. Malin and S. Woods, «Efficient high-power diode LASER cladding - Industrial LASER Solutions». [Online]. Available: http://www.industrial-lasers.com/articles/print/volume-24/issue8/features/application-report/efficient-high-power-diode-laser-cladding.html. [Accessed: 02-Jan-2017].

[13] E. Lugscheider, H. Bolender, and H. Krappitz, «LASER Cladding of Paste Bound Hardfacing Alloys», Surf. Eng., vol. 7, n. 4, pp. 341-344, 1991.

[14] J. M. S. P. Torres, T. L. M. Morgado, and H. V. G. Navas, «Innovative Automation Equipment for LASER Cladding», presented at The 2015 International Conference on Systematic Innovation, Hong Kong, 2015.

[15] T. L. M. Morgado, H. Navas, and R. Brites, «Wear study of Innovative Ti-Ta alloys», Procedia Struct. Integr., vol. 2, pp. 1266-1276, 2016.

[16] H. I. M. Souto, «LASER Cladding: its application to the deposition of coatings in blades of wood crushers» in Portuguese, Master Thesis, FEUP, UP - Universidade do Porto, Portugal, 2013.

[17] K. Feng et al., «Improved high-temperature hardness and wear resistance of Inconel 625 coatings fabricated by LASER cladding», J. Mater. Process. Technol., vol. 243, pp. 82-91, 2017.

[18] S. R. Lewis et al., «Improving rail wear and RCF performance using LASER cladding», Wear, vol. 366-367, pp. 268-278, 2016.

[19] S. Da Sun, M. Leary, Q. Liu, and M. Brandt, «Evaluation of microstructure and fatigue properties in LASER cladding repair of ultrahigh strength AerMet ${ }^{\circledR} 100$ steel», J. Laser Appl., vol. 27, n. S2, pp. S29202-1 - S29202-6, 2015.

[20] J. Tuominen, «Engineering Coatings by LASER Cladding - The Study of Wear and Corrosion Properties», Ph.D. Dissertation, Tampereen teknillinen yliopisto, Suomi, 2009. 
[21] A. D. Ludovico, A. Angelastro, e S. L. Campanelli, «Experimental Analysis of the Direct LASER Metal Deposition Process», in New Trends in Technologies: Devices, Computer, Communication and Industrial Systems, Sciyo, 2010, ch.14, pp. 253-272.

[22] T. M. Yue, Y. P. Su, and H. O. Yang, «LASER cladding of Zr65Al7.5Ni10Cu17.5 amorphous alloy on magnesium», Mater. Lett., vol. 61, n. 1, pp. 209-212, 2007.

[23] Q. Meng, L. Geng, and D. Ni, «LASER cladding NiCoCrAlY coating on Ti-6Al-4V», Mater. Lett., vol. 59, n. 22, pp. 2774-2777, 2005. 